PROCEEDINGS OF THE AMERICAN MATHEMATICAL SOCIETY

Volume 124, Number 5, May 1996

\title{
DEGREE OF IRRATIONALITY OF A PRODUCT OF TWO ELLIPTIC CURVES
}

\author{
HISAO YOSHIHARA
}

(Communicated by Eric M. Friedlander)

\begin{abstract}
We consider the degree of irrationality $d_{r}(S)$ of some algebraic surface $S$. Firstly we give an estimate of $d_{r}(S)$ for a surface $S$ with a structure of a fiber space. Secondly we prove the existence of a nonsingular curve of genus 3 on $E \times E$ for a certain elliptic curve $E$ with complex multiplications. As a corollary, we obtain that $d_{r}(E \times E)=3$.
\end{abstract}

\section{InTRODUCTION}

Let $V$ be an $n$-dimensional algebraic variety defined over a field $k$, and let $k(V)$ be the rational function field of $V$. The degree of irrationality of $V$ is defined to be the least number $m$ such that $m=\left[k(V): k\left(x_{1}, \ldots, x_{n}\right)\right]$, where $x_{1}, \ldots, x_{n}$ are algebraically independent elements of $k(V)$ (cf. [6], [9]). By definition this number is a birational invariant and we denote it by $d_{r}(V)$. In other words it is the minimal degree of a dominant rational map from $V$ to the projective $n$-space. In the case when $n=1, d_{r}(V)$ coincides with the gonality of a curve and has been studied mainly for plane curves (see, e.g., [3]).

In what follows we assume that $k=\mathbf{C}$ and we work in the category of algebraic varieties over $\mathbf{C}$. When $n=2$ and $d_{r}(V)=2$, some results are obtained in [8]. For an abelian variety $A$, it is proved that $d_{r}(A) \geq n+1$ in [1]. Clearly we have that $d_{r}(A)=2$ if $n=1$. It seems to be important to determine the value $d_{r}(A)$ when $n=2$, but only a few results have been obtained; for example, if $A$ is a double covering of a Jacobian variety of a curve, then $d_{r}(A)=3$ (see [7]). In this paper we will give an estimate of $d_{r}$ for a surface with a structure of a fiber space and prove the existence of a nonsingular curve of genus 3 on $E \times E$, where $E$ is a certain elliptic curve with complex multiplications. As a corollary, we obtain that $d_{r}(E \times E)=3$.

\section{Statement of Results}

First we present an estimate of $d_{r}$ for a surface with a structure of a fiber space.

Proposition 1 (cf. [7]). Let $S$ and $C$ be a nonsingular projective surface and curve, respectively. Suppose that there is a surjective morphism $f: S \rightarrow C$, whose general

Received by the editors October 13, 1994.

1991 Mathematics Subject Classification. Primary 14J25; Secondary 14K99.

Key words and phrases. Degree of irrationality, abelian surface, elliptic curve with complex multiplications. 
fiber $F$ is irreducible. Let $\mathrm{g}(F)$ denote the genus of $F$. Then we have the following assertions:

(1) If $\mathrm{g}(F)=0$, then $d_{r}(S)=d_{r}(C)$.

(2) If $\mathrm{g}(F)=1$ and $f$ has a section, then $d_{r}(S) \leq 2 d_{r}(C)$.

(3) If $\mathrm{g}(F) \geq 2$ and $d_{r}(F)=2$, then $d_{r}(S) \leq 2 d_{r}(C)$.

(4) If $\mathrm{g}(F)=3, d_{r}(F) \neq 2$ and $f$ has a section, then $d_{r}(S) \leq 3 d_{r}(C)$.

If we drop the assumption that $f$ has a section in (2), then the conclusion does not hold true. For example let $S$ be a hyperelliptic surface; then it has a structure of an elliptic fiber space with multiple singular fibers. We can shown that $d_{r}(S) \geq 3$ for some $S$. The case (4) has been proved by a different method in [7]. Using this proposition, we get

Theorem 2. If there is a nonsingular curve of genus 3 on an abelian surface $A$, then $d_{r}(A)=3$.

Note that if there is a nonsingular curve $C$ of genus 2 on $A$, then $A$ is a Jacobian variety $J(C)$ of $C$. If an abelian surface $B$ is a double covering of $J(C)$, then $d_{r}(B)=3$ by this theorem.

In the sequel we use the following notation. Let $m$ be a positive square free integer. Put $\omega=\sqrt{-m}$ [resp. $\frac{1+\sqrt{-m}}{2}$ ] if $-m \equiv 2$ or $3 \quad(\bmod 4)[$ resp. $-m \equiv 1$ $(\bmod 4)]$. Let $K=\mathbf{Q}(\sqrt{-m})$ be an imaginary quadratic field. For each $\xi \in K \backslash \mathbf{Q}$, let $a \xi^{2}+b \xi+c=0$ be the equation for $\xi$ with $a, b, c \in \mathbf{Z}, a>0$ and $(a, b, c)=1$. Let $L$ be the lattice generated by $\{1, \xi\}$ and let $E$ be the elliptic curve $\mathbf{C} / L$.

Theorem 3. Under the situation above, suppose that at least one of $a, b, c$ is an even number. Then there exist two elliptic curves $E_{1}$ and $E_{2}$ on $A=E \times E$ satisfying $\left(E_{1}, E_{2}\right)=2$, where $\left(E_{1}, E_{2}\right)$ denotes the intersection number of $E_{1}$ and $E_{2}$. Especially there exists a nonsingular curve of genus 3 on $A$, hence $d_{r}(A)=3$.

Note 4. Of course there are many elliptic curves $E$ satisfying the condition in Theorem 3. In fact, if $-m \equiv 2$ or $3(\bmod 4)$, then $b$ is even, because $a \xi$ becomes an integer. Hence every $\xi$ enjoys the condition. For the remaining case, letting $k$ and $l(\neq 0)$ be rational integers, we have the following.

(i) If $-m \equiv 1(\bmod 8)$, then $\xi=k+l \omega$ and $\frac{1}{2}+l \omega$ are the suitable ones.

(ii) If $-m \equiv 5(\bmod 8)$, then $\xi=k+2 l \omega$ and $\frac{1}{2}+l \omega$ are the suitable ones.

However we notice the following assertion.

Proposition 5. Suppose that $\xi=\omega$ and $m=3,11,19,43,67$ or 163 . Then there exist no elliptic curves $E_{1}$ and $E_{2}$ satisfying $\left(E_{1}, E_{2}\right)=2$ on $E \times E$.

Remark 6. When $m=3$ and $\xi=\omega$, we consider the quotient of $E \times E$ by the automorphism $\left(z_{1}, z_{2}\right) \mapsto\left(\omega z_{1}, \omega z_{2}\right)$. Then the quotient space turns out to be a rational surface and hence $d_{r}(E \times E)=3$ (cf. [7]).

Example 7. As an application of Theorem 2, we take an example from $[2,(1.8)]$. Let $D$ be a nonsingular curve of genus 3 which admits an elliptic involution $\pi: D \rightarrow$ $E$. Let $e_{0} \in D$ be a branchpoint for $\pi$ and embed $D \rightarrow J=\operatorname{Pic}^{0}(D)$ via $p \mapsto$ $\mathcal{O}_{D}\left(p-e_{0}\right)$. By using $x_{0}=\pi e_{0}$, we identity $E \rightarrow \operatorname{Pic}^{0}(E)$ via $x \mapsto \mathcal{O}_{E}\left(x-x_{0}\right)$. Then we have a natural injection $\pi^{*}: E \rightarrow J$. We put $A=J / \pi^{*} E$; then the map $D \hookrightarrow J \rightarrow A$ turns into an embedding. Hence we have $d_{r}(A)=3$. 


\section{Proof}

First we prove Proposition 1. Let us treat the case (1). Since $S$ is birationally equivalent to $C \times \mathbf{P}^{1}$, we have that $d_{r}(S)=d_{r}\left(C \times \mathbf{P}^{1}\right)$. Then we get $d_{r}\left(C \times \mathbf{P}^{1}\right)=$ $d_{r}(C)$ (cf. [9]). Proofs of (2), (3) and (4) are done simultaneously. Let $\mathcal{K}_{S}$ denote the canonical divisor on $S$ and let $\Gamma$ be the section in (2) and (4). Let $\mathcal{F}$ be the sheaf on $S$ equal to $\mathcal{O}_{S}(2 \Gamma), \mathcal{O}_{S}\left(\mathcal{K}_{S}+F\right)$ and $\mathcal{O}_{S}\left(\mathcal{K}_{S}-\Gamma\right)$, corresponding to the cases (2), (3) and (4) respectively. Since $f_{*} \mathcal{F}$ is a coherent sheaf on $C$, we have a projective fiber space $\mathbf{P}\left(f_{*} \mathcal{F}\right) \rightarrow C$ associated with $f_{*} \mathcal{F}$ and a rational map $g: S-\rightarrow \mathbf{P}\left(f_{*} \mathcal{F}\right)$. Let $X$ be the image of $g$. Then we see that $X$ is a ruled surface over $C$. In the case (2) or (3), the degree of $g$ is 2 , hence we conclude that $d_{r}(S) \leq 2 d_{r}(C)$ by (1). On the other hand, in the case (4), since

$$
\operatorname{dim} H^{0}\left(F, \mathcal{O}\left(\mathcal{K}_{F}-F \cap \Gamma\right)\right)=h^{0}\left(F, \mathcal{O}\left(\mathcal{K}_{F}-F \cap \Gamma\right)\right)=2
$$

for a general fiber $F$ and it is not hyperelliptic, the rational map $g$ has degree 3 . Hence, similarly we infer that $d_{r}(S) \leq 3 d_{r}(C)$.

Next we prove Theorem 2. Let $C$ be the nonsingular curve of genus 3 . Since $C^{2}=(C, C)=4$, we see that $C$ is ample and $h^{0}(A, \mathcal{O}(C))=2$ from the RiemannRoch theorem. The rational map defined by the complete linear system $|C|$ has 4 base points. By blowing-up these points, we get a morphism $f: \widetilde{A} \rightarrow \mathbf{P}^{1}$. Clearly $f$ has 4 sections. As we mentioned in the Introduction, we have that $d_{r}(A) \geq 3$, hence it is sufficient to show that a general fiber is not hyperelliptic. Suppose that except for finitely many fibers every fiber is hyperelliptic. Then we have $d_{r}(A)=2$ by (3), which is a contradiction. Hence a general fiber must be non-hyperelliptic, because in the moduli space of curves of genus 3 , hyperelliptic ones consist of an analytic subspace. Thus by (4) we obtain $d_{r}(A)=3$.

Before the proof of Theorem 3 we provide two lemmas. The next one may be well-known.

Lemma 8. Let $E$ be an elliptic curve on an abelian surface $A$. Then $h^{0}(A, \mathcal{O}(E))=$ 1.

Proof. From the exact sequence of sheaves

$$
0 \rightarrow \mathcal{O}_{A}(-E) \rightarrow \mathcal{O}_{A} \rightarrow \mathcal{O}_{E} \rightarrow 0
$$

we get the long exact sequence of cohomology groups

$$
\begin{aligned}
0 \rightarrow H^{1}(A, \mathcal{O}(-E)) \rightarrow H^{1}\left(A, \mathcal{O}_{A}\right) \stackrel{r}{\rightarrow} H^{1}\left(E, \mathcal{O}_{E}\right) & \\
& \rightarrow H^{2}(A, \mathcal{O}(-E)) \rightarrow H^{2}\left(A, \mathcal{O}_{A}\right) \rightarrow 0 .
\end{aligned}
$$

From this sequence and by the Serre duality theorem, we infer that $h^{0}(A, \mathcal{O}(E))=$ $\operatorname{dim} H^{1}(A, \mathcal{O}(-E))=h^{1}(A, \mathcal{O}(-E))$. On the other hand, referring to [4, p. 571], we see that $\operatorname{dim} \operatorname{ker} r$ is the number of linearly independent holomorphic 1-forms on $A$ which vanish on $E$. Whence we have that $h^{1}(A, \mathcal{O}(-E)) \leq 1$, which proves the assertion.

Lemma 9. If there are two elliptic curves $E_{1}$ and $E_{2}$ satisfying $\left(E_{1}, E_{2}\right)=2$ on an abelian surface $A$, then there is a nonsingular curve of genus 3 on $A$.

Proof. Putting $D=E_{1}+E_{2}$, we see that $D$ is an ample divisor and hence $h^{0}(A, \mathcal{O}(D))=2$. By the above lemma the pencil $|D|$ has no fixed component. Hence by Bertini's theorem its general member is an irreducible nonsingular curve of genus 3 (cf. $[2,(1.4)]$ ). 
Now we proceed to the proof of Theorem 3. Let $\varphi_{\alpha, \beta}: E \rightarrow E \times E$ be a morphism defined by $\varphi_{\alpha, \beta}(z)=(\alpha z, \beta z)$, where $\alpha$ and $\beta \in \operatorname{End}(E)$. Note that $\operatorname{End}(E)$ is generated by 1 and $a \xi$ over $\mathbf{Z}$. Put $E_{\alpha, \beta}=\varphi_{\alpha, \beta}(E)$. By taking a suitable $(\alpha, \beta ; \gamma, \delta)$, we may obtain that $\left(E_{\alpha, \beta}, E_{\gamma, \delta}\right)=2$. For example $\left(E_{0,1}, E_{2, \lambda}\right)=2$ if we take $\lambda$ as follows: In case $a$ is even, let $\lambda=a \xi$. On the contrary, in case $a$ is odd, let $\lambda=x+y(a \xi)$, where $x$ and $y(\neq 0)$ are given as follows: If $b$ and $c$ are even, then let $x$ be even and $y$ be odd. If $b$ or $c$ is odd, then let $x$ and $y$ be odd. By simple calculations we see that the number of the elements of the set $\left\{(2 z, \lambda z) \in E_{2, \lambda} \mid 2 z=0\right.$ in $\left.E\right\}$ is 2 . Since $E_{0,1}$ and $E_{2, \lambda}$ meet transversally, we have that $\left(E_{0,1}, E_{2, \lambda}\right)=2$. Using Lemma 9, we finish the proof of Theorem 3 .

Next we prove Proposition 5. Since $\operatorname{End}\left(E_{i}\right)$ becomes a maximal order of $K$ in this case, we make use of the results of [5]. Suppose that such curves $E_{i}(i=1,2)$ exist. Then $E_{i}$ is a translation of $E_{\alpha_{i}, \beta_{i}}$ for some $\alpha_{i}, \beta_{i} \in \operatorname{End}(E)$ (cf. [5, Lemma 1]). Hence

$$
\left(E_{\alpha_{1}, \beta_{1}}, E_{\alpha_{2}, \beta_{2}}\right)=\left(E_{1}, E_{2}\right)=2 .
$$

Moreover, by [5, Corollary 1 on p. 6], we have that

$$
\left(E_{\alpha_{1}, \beta_{1}}, E_{\alpha_{2}, \beta_{2}}\right)=\frac{N\left(\alpha_{1} \beta_{2}-\alpha_{2} \beta_{1}\right)}{N\left(\alpha_{1}, \beta_{1}\right) N\left(\alpha_{2}, \beta_{2}\right)},
$$

where $N$ denotes the norm. Clearly we also have that

$$
\left(E_{\bar{\alpha}_{1} \alpha_{1}, \bar{\alpha}_{1} \beta_{1}}, E_{\bar{\alpha}_{2} \alpha_{2}, \bar{\alpha}_{2} \beta_{2}}\right)=2 .
$$

We can write $\bar{\alpha}_{i} \alpha_{i}=c_{i} a_{i}, \bar{\alpha}_{i} \beta_{i}=c_{i} b_{i}+c_{i} \omega$, where $a_{i}, b_{i}, c_{i} \in \mathbf{Z}(i=1,2)$ and we may assume that $\left(c_{i} a_{i}, c_{i} b_{i}+c_{i} \omega\right)$ form a canonical basis. Then we infer from the above that $\gamma \bar{\gamma}=2 a_{1} a_{2}$, where $\gamma=\left(a_{1} b_{2}-b_{1} a_{2}\right)+\left(a_{1}-a_{2}\right) \omega$. Since 2 is a prime number in $K$ and the class number of $K$ is 1 , we see that $a_{1}$ and $a_{2}$ are even numbers. Putting $a_{i}=2 a_{i}^{\prime}$, we obtain that $\gamma^{\prime} \bar{\gamma}^{\prime}=2 a_{1}^{\prime} a_{2}^{\prime}$, where $\gamma^{\prime}=\left(a_{1}^{\prime} b_{2}-b_{1} a_{2}^{\prime}\right)+\left(a_{1}^{\prime}-a_{2}^{\prime}\right) \omega$. We can repeat the same argument finitely many times, which gives rise to a contradiction.

Finally we mention a problem concerning $d_{r}$.

Problem 10. Find the value $d_{r}(A)$ for each abelian surface $A$. Especially we ask whether the following assertions hold true:

(1) Is there an abelian surface $A$ satisfying $d_{r}(A) \geq 4$ ? For example, is it true that $d_{r}\left(E_{1} \times E_{2}\right)=4$ if $E_{1}$ and $E_{2}$ are not isogenous?

(2) If two abelian surfaces $A_{1}$ and $A_{2}$ are isogenous, then is it true that $d_{r}\left(A_{1}\right)=$ $d_{r}\left(A_{2}\right) ?$

\section{REFERENCES}

[1] A. Alzati and G. P. Pirola, On the holomorphic length of a complex projective variety, Arch. Math. 59 (1992), 398-402. MR 94b:14014

[2] W. Barth, Abelian surfaces with $(1,2)$-polarization, Advanced Studies in Pure Math. 10 (1987), 41-84. MR 89i:14038

[3] M. Coppens and T. Kato, The gonality of smooth curves with plane models, Manuscripta Math. 70 (1990), 5-25. MR 92a:14027a

[4] P. Griffiths and J. Harris, Principles of Algebraic Geometry, New York 1978. MR 80b:14001

[5] T. Hayashida and M. Nishi, Existence of curves of genus two on a product of two elliptic curves, J. Math. Soc. Japan 17 (1965), 1-16. MR 34:1318

[6] T. T. Moh and W. Heinzer, On the Lüroth semigroups and Weierstrass canonical divisors, J. Algebra 77 (1982), 62-73. MR 83k:14027 
[7] H. Tokunaga and H. Yoshihara, Degree of irrationality of abelian surfaces, J. Algebra 174 (1995), 1111-1121. CMP 95:14

[8] H. Yoshihara, Double coverings of rational surface, Manuscr. Math. 75 (1992), 279-291. MR 93d:14055

[9] H. Yoshihara, Degree of irrationality of an algebraic surface, J. Algebra, 167 (1994), 634-640. MR 95g:14039

Department of Mathematics, Faculty of Science, Niigata University, 950-21 Niigata, JAPAN

E-mail address: yosihara@geb.ge.niigata-u.ac.jp 